\title{
Epidemiología de la infección y detección de tipos oncogénicos del VPH por captura de híbridos en mujeres sin factores de riesgo aparentes
}

\section{Epidemiology of Infection and Detection of Oncogenic Types of Human Papillo- ma Virus (HPV) by Hybrid Capture in Women without Apparent Risk Factors}

\author{
David de la Fuente Villarreal ${ }^{1}$, Santos Guzman Lopez ${ }^{1}$, Arnulfo Gomez Sanchez ${ }^{1}$, \\ Bernardo Fernandez Rodarte ${ }^{1}$, David A. Martinez Fernandez ${ }^{1}$. Pedro T. Cortes González ${ }^{2}$ \\ 1 Depto. de Anatomía Humana de la Facultad de Medicina de la UANL. \\ 2 Servicio de Neurocirugía del Hospital Universitario “Dr. Jose Eleuterio González" \\ Correspondencia: Dr. David de la Fuente Villarreal, Correo electrónico: drdelafuentev@gmail.com
}

Fecha de recepción: Julio 10-2013

Fecha de aceptación: Diciembre 28-2013

De la Fuente-Villarreal D, Guzmán S, Gómez A, et al. Epidemiology of Infection and Detection of Oncogenic Types of Human Papilloma Virus (HPV) by Hybrid Capture in Women without Apparent Risk Factors. RFS - Revista Facultad de Salud. 2013; 5 (2): 34-40.

\section{Resumen}

Objetivo: Determinar la prevalencia de infección por el virus del papiloma humano (VPH) en la población femenina, sin aparentes factores de riesgo, que espontáneamente acude a realizarse la prueba de Papanicolaou en el Hospital Universitario Dr. José E. González durante el periodo 2006 - 2010.

Material y métodos: Se realizó un estudio observacional, transversal, prospectivo, analítico comparativo en 361 pacientes, las cuales espontáneamente solicitaron una prueba de citología vaginal.

Resultados: Se estudiaron un total de 361 mujeres, de las cuales se eliminaron 18 (5\%) por no haberse obtenido muestra suficiente, quedando una muestra final significativa de 343 pacientes, demostrando infección por VPH de cualquier tipo en 51 pacientes (14,9\%), se observó mayor prevalencia en el grupo de divorciadas positivas $(11,8 \%)$, con respecto al de divorciadas negativas del $3,1 \%,(p=0,0152)$, se estableció una correlación significativa $(p=0,001)$ entre el número de parejas sexuales y la positividad de la prueba $(R h o=0,175)$, así como una asociación significativa entre la característica de ser divorciada y ser positiva $(p=0,0152, X 2=5,888)$.

Discusión: La exploración ginecológica normal no descarta la presencia de VPH e igualmente, una citología normal o con cambios inflamatorios inespecíficos, tampoco permite concluir la ausencia de VPH, de tal manera que la detección temprana de infección por VPH requiere métodos moleculares de diagnóstico. Podemos concluir que con los métodos tradicionales de diagnóstico, no es posible detectar todas las pacientes infectadas con $\mathrm{VPH}$, por lo cual sería deseable practicar pruebas moleculares para diagnóstico de la infección. 
Palabras clave: cáncer cervical, Virus del Papiloma Humano, prevalencia, tamizaje

\begin{abstract}
Objective: to determine prevalence of infection by human papilloma virus (HPV), in female population, with no apparent risk factors, who spontaneously undergo the Papanicolaou test at the Hospital Universitario Dr. José E. González during the 2006-2010 period.
\end{abstract}

Materials and methods: An observational, cross-sectional, prospective, analytic, comparative study in 361 patients who spontaneously requested a vaginal cytology test.

Results: A total of 361 women were studied from which 18 (5\%) were taken out since their sample was not enough, thereby resulting in a final significant sample of 343 patients. The study showed infection by HPV in 51 patients $(14,9 \%)$; there was higher prevalence in the positive divorced group $(11,8 \%)$; with respect to the negative divorced group $3,1 \%(p=0.0152)$. It was established a significant correlation $(p=0,001)$ between the number of sexual partners and the test positivity $(R h o=0,175)$, as well as a significant association between being divorced and being positive $(p=0,0152, X 2=5,888)$.

Discussion: normal gynecological examination does not rule out the presence of VPH. Likewise, a normal vaginal cytology report or with non-specific inflammatory changes does not allow to conclude the absence of HPV, so that early detection of infection by HPV, calls for molecular methods of diagnosis. It can be concluded that by using traditional methods of diagnosis it is not possible to detect all HPV-infected patients and it would be desirable to perform molecular tests for infection diagnosis.

Keywords: cervical cancer, human papilloma virus, prevalence, screening.

\section{Introducción}

El cáncer cervicouterino (CaCU) es una de las principales neoplasias malignas que atacan a la población femenina mundial y en México es la causa más frecuente de mortalidad por cáncer. ${ }^{(1)}$ Está bien establecida la asociación epidemiológica entre este cáncer y el inicio temprano de la actividad sexual y con la presencia de múltiples parejas sexuales. Para el año 2000 se estableció un total de 470.606 casos nuevos y 233.372 defunciones asociadas al CaCU, Zhang WY et al. (2008) determinaron en 6405 pacientes una prevalencia del $29.1 \%{ }^{(2)}$.

El virus del papiloma humano (VPH) es considerado como el principal factor asociado para el desarrollo de CaCU, existiendo un relación directa entre la incidencia de cáncer cervicouterino y la prevalencia de VPH entre la población ${ }^{(7)}$; dichos hallazgos fueron establecidos Inicialmente en Maracaibo en $1971^{(8)}$, pero no fue hasta 1977 que ZurHausen sugirió que podía existir asociación entre el VPH y el cáncer cer- vical. ${ }^{(9)}$ Durante esta década, se describieron los modelos de carcinogénesis inducida por virus en humanos, en pacientes con carcinomas escamosos cutáneos originados en epidermodisplasia verruciforme, enfermedad causada por un tipo de VPH. ${ }^{(10,11)}$

La epidemiología de la infección por VPH difiere para cada población, ya que el resultado depende de diversas variables, el estilo de vida, parejas sexuales, inicio de vida sexual presencia o ausencia de manifestaciones clínicas y la sensibilidad y especificidad del método diagnóstico principalmente. La prevalencia del VPH en países con una alta frecuencia de CaCU se estima entre el $10 \%$ y $20 \%$, mientras que en países de baja frecuencia es del 5\% al 10\%; Six L, Leodolter S, Sings HL, et al. (2008) encontraron en 123 pacientes una prevalencia de $13.5 \%$ para el VPH 6, 11, 16 o 18 por PCR; serológicamente el 12,3\% de las mujeres fueron positivos para VPH 6, 11, 16 o 18, de ello 11,4\% fueron positivos para VPH 16, 3,5\% para VPH 
18, y 0,9\% para el VPH $6{ }^{(3)}$; en América latina se estimaron 92.136 casos y 37.640 defunciones por $\mathrm{CaCU}$, de los cuales el $83,9 \%$ y el $81,2 \%$ corresponderían a América Latina y el Caribe, respectivamente. ${ }^{(4)}$

En México anualmente se estima un total de 4.000 muertes por $\mathrm{CaCU}$, en el 2005 se registraron 4.270 casos con una tasa de 10,2 por 100.000 mujeres, para el año 2009 esta tasa se incrementó a 19,3 casos por cada 100.000 mujeres $^{(5)}$, siendo el grupo etario más afectado por los serotipos $16,18,31,33,35,45,51,52,58$ y 59 el de 35 a 64 años, representando la primera causa de mortalidad en mujeres. ${ }^{(6)}$

En un estudio realizado en jóvenes mexicanos con dos o más parejas sexuales, Sánchez-Alemán et al. determinaron una prevalencia de VPH del 14,4\%. ${ }^{(12)}$ Determinaron que el curso de la infección depende primordialmente del tipo de VPH encontrado, la edad de adquisición y del estado inmune de la paciente ${ }^{(13)}$; establecieron que las mujeres menores de 35 años son más susceptibles a adquirir infecciones genitales con virus no oncogénicos (y en la mayoría de los casos dicha infección desaparece) $)^{(14)}$; en cambio, en las mujeres mayores de 35 años es más común la persistencia de la lesión con cambios clínicos y morfológicos y con mayor riesgo de progresión neoplásica. ${ }^{(15,16) .}$

Este estudio muestra la prevalencia de VPH en una población mexicana y analiza su correlación entre variables relevantes como el número de parejas sexuales.

\section{Material y métodos.}

Se realizó un estudio observacional, trasversal, prospectivo, analítico comparativo en 361 pacientes, las cuales espontáneamente solicitaron una prueba de citología vaginal en el módulo de detección oportuna de cáncer (D.O.C.), del servicio de Ginecología, en el Hospital Universitario Dr. José E. González de la UANL. de enero de 2006 hasta mayo de 2010.
El personal de salud adscrito al módulo de D.O.C., del Hospital Universitario Dr. José E. González de la UANL. previamente capacitado, obtuvo las muestras de la zona de transición del cérvix uterino con un pequeño cepillo cónico rotándolo tres veces, después de lo cual se colocó en un tubo de $5 \mathrm{ml}$ con el medio de transporte de citología de base líquida, estas fueron evaluadas mediante las técnicas de Papanicolaou y de captura de híbridos de segunda generación o HC2 ${ }^{\circledR}$ con una sensibilidad y especificidad de 93,3\% (95\%IC: 92,5-94) y 89,2 \% (95\%IC: $88,3-$ $90,1)$ respectivamente para la identificación y serotipificación de lo subtipos de VPH de bajo riesgo carcinogénico $(6,11,42,43$ y 44) y el grupo de subtipos de VPH de alto riesgo carcinogénicos $(16,18,31,33,35,39,45,51,52,56,58,59 \mathrm{y}$ 68) a un nivel de $1 \mathrm{pg} / \mathrm{ml}$ cada uno, que corresponde a 5,900 genomas de VPH cada prueba a fin de determinar la presencia de la infección.

Los resultados de las variables analizadas (Identificación del paciente, edad, estado civil, edad de inicio de vida sexual activa (IVSA), número de compañeros sexuales, fecha del último Papanicolaou, resultado del Papanicolaou, Papanicolaou subsecuente, situación ginecológica, exploración clínica, diagnóstico citológico, captura de híbridos para establecer la presencia de tipos del VPH de riesgo alto y riesgo bajo) fueron analizados mediante el programa SPSS en su versión 12, los estadísticos descriptivos tradicionales de tendencia central, de dispersión y de posición en el caso de las variables cuantitativas; en las variables cualitativas se determinaron las frecuencias observadas. Los sujetos de estudio se dividieron según la presencia o ausencia de positividad mediante la prueba de captura de híbridos, posteriormente se contrastaron mediante pruebas de hipótesis para medias y proporciones, según sea el caso para cada tipo de variable, a una confiabilidad del 95\%. La de correlación o asociación entre la positividad y los factores evaluados se realizó mediante la prueba de Correlación de Pearson o Spearman y $X^{2}$ (según sea el caso) a una confianza del 95\%. 
Todas las pacientes que participaron expresaron su libre voluntad de participar mediante la firma del consentimiento informado, prevaleciendo el criterio de respeto, dignidad y confidencialidad en los derechos de los pacientes, de acuerdo a los principios de la declaración de Helsinki, y con la Ley General de Salud, Título Segundo, de los aspectos Éticos de la investigación en Seres Humanos Capítulo I, disposiciones comunes artículo 13 y 14.

\section{Resultados}

Se estudiaron un total de 361 mujeres, de las cuales se descartaron 18 (5\%) por no haberse obtenido muestra suficiente, quedando una muestra final significativa de 343 pacientes, demostrando infección por VPH de cualquier tipo en 51 pacientes $(14,9 \%)$. La edad promedio de las pacientes fue de 40,0 años, siendo los 40 años cumplidos, la edad más observada y el valor máximo registrado en el 50\% de las pacientes; estos datos presentan una variabilidad con respecto al promedio de 12,1 años (Figura 1).

La variable IVSA (edad de inicio de la vida sexual activa) mostró una media de 19,9 años, siendo los 18 años el valor más frecuente referido en este rubro; la mitad de las pacientes dijeron haber iniciado su vida sexual a una edad igual o inferior a los 19 años, con una variabilidad con respecto al promedio de 3,8 años.

Al cuestionar el resultado de su último Papanicolaou anterior al inicio de nuestro estudio, el $75,8 \%$ de ellas recordaban que éste fue negativo, la situación ginecológica de las pacientes en el momento del estudio era aparentemente normal en el $87,5 \%$ de los casos y en el restante $14,6 \%$ de las pacientes se presentaron alteraciones mostradas en la figura 1; al evaluar los hallazgos adicionales en las pacientes evaluadas, estos solo se presentaron en el 14,6\% de los estudios realizados, comportándose como se muestra en la (figura 2).

Figura 1. Distribución porcentual, según el resultado de la exploración ginecológica del cuello del útero. $n=343$ pacientes/51 pacientes positivas.

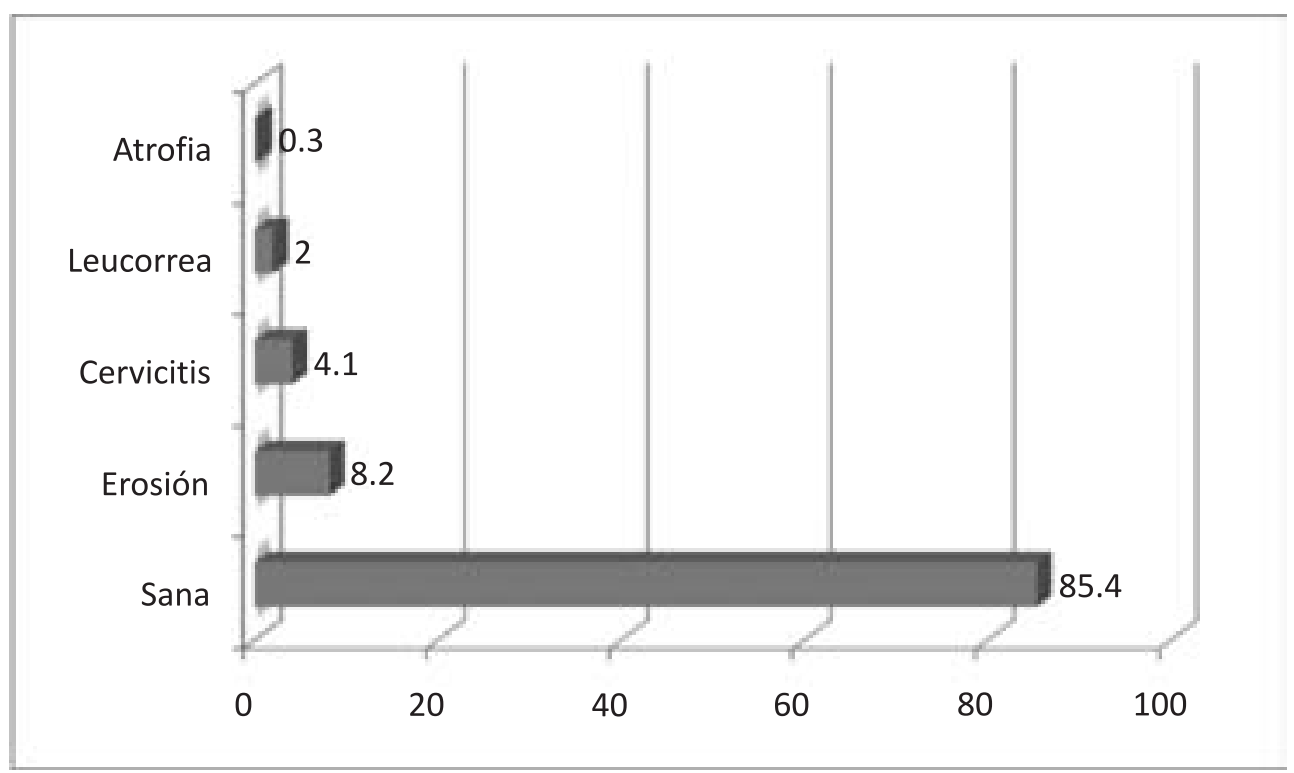

n=343 pacientes/51 pacientes positivas. / Fuente: Historia clínica. 
Figura 2. Distribución porcentual de los hallazgos adicionales.

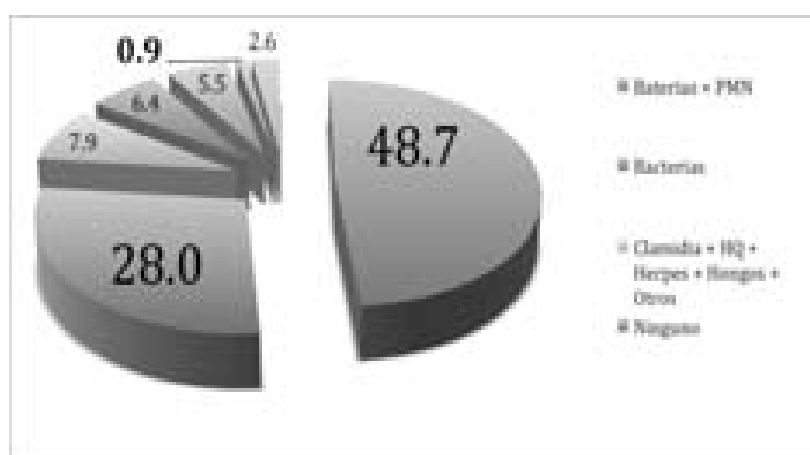

$\mathrm{n}=343$ pacientes $/ 51$ pacientes positivas. / Fuente: Historia clínica.

Con respecto a la exploración ginecológica, el $82 \%(42 / 51)$ de las pacientes infectadas no mostró ninguna alteración. Sin embargo, 4 mujeres presentaron leucorrea, y en 1 se diagnosticó cervicitis (10\%); además, en 4 pacientes se observaron erosiones $(8 \%)$.

De otra parte, en la valoración de los hallazgos citológicos de las pacientes infectadas, se encontró un $4 \%$ con citología negativa; en el $66 \%$, sólo se describieron cambios inflamatorios inespecíficos; el 18\% presentó células escamosas atípicas de significado incierto (ASC-US); el 6\% mostró lesión intraepitelial de bajo grado (LIEBG); $\mathrm{y}$, finalmente, en el $6 \%$ de las pacientes $\mathrm{VPH}+$ se diagnosticó una lesión intraepitelial de alto grado (LIEAG).

Se compararon todas la variables antes mencionadas, mediante pruebas de hipótesis para medias y proporciones en el caso de variables cuantitativas y cualitativas, respectivamente, a una confiabilidad del 95\% y utilizando como constante de segmentación la positividad por la prueba de híbridos para VPH. Se encontraron diferencias estadísticamente significativas entre el promedio de parejas sexuales $(p=0,0008)$ de las pacientes negativas (1,25 parejas) y el de las positivas (1,47 parejas).

Al analizar el estado civil de las pacientes, se observó mayor prevalencia de la patología es- tudiada entre las mujeres divorciadas con resultado positivo $(11,8 \%)$, en contraste con las mujeres divorciadas con resultado negativo $(3,1 \%)$, siendo esta diferencia estadísticamente significativas $(p=0,0152)$. Al realizar las pruebas de correlación de Spearman y $\mathrm{X}^{2}$ a una confiabilidad del 95\%, pudo establecerse una correlación estadísticamente significativa $(p=0,001)$ entre el número de parejas sexuales y la positividad de la prueba (Rho=0,175), así como una asociación significativa entre la característica de ser divorciada y ser positiva $\left(p=0,0152, X^{2}=5,888\right)$. No se encontraron otros cruces estadísticamente significativos mediante las pruebas y condiciones antes mencionadas.

\section{Discusión}

Actualmente vivimos una etapa que podríamos considerar como histórica en relación con el control del cáncer cervical, si consideramos el nuevo paradigma para la prevención primaria y secundaria de la enfermedad.

Hemos tardado 20 años en establecer la asociación causal entre el cáncer cervical y la infección persistente por una enfermedad de transmisión sexual, el virus de papiloma humano o VPH. Actualmente, la historia natural de la infección por VPH y el cáncer cervical es ampliamente conocida y, por ello, se han desarrollado pruebas de detección del ADN del VPH de alto riesgo para mejorar la eficiencia, precisión y efectividad de la detección oportuna de cáncer, como estrategia de prevención secundaria; muchos países desarrollados utilizan actualmente esta estrategia de prevención con programas poblacionales de detección primaria de lesiones precursoras de cáncer.

En nuestro estudio, se observó que 14,9 mujeres de cada 100 sin aparentes factores de riesgo presentan infección por VPH, prevalencia que es equiparable a la publicada previamente y obtenida de una población de jóvenes universitarias mexicanas. 
La prevalencia de la infección por VPH detectada en nuestra población es muy inferior a la publicada por las series americanas, que van del $26,8 \%$ en la población general de 14 a 59 años, hasta el $44,8 \%$ en el grupo de 20 a 24 años de edad; lo cual podría explicarse por la diferente cultura referente a la sexualidad, entre ambas poblaciones. El estudio de Zhang et al. muestra una prevalencia de infección por VPH ligeramente superior a la reportada por nosotros, ya que a través de un estudio prospectivo encontraron una prevalencia del 29,1\% de VPH de alto riesgo oncogénico en una extensa población de mujeres sexualmente activas $(n=6405$, con un rango de edad de 18-78 años), siendo el subgrupo de 18-20 años de edad, el de la prevalencia más elevada $(54,4 \%)$, detectados por medio de captura de híbridos de segunda generación $\left(\mathrm{HC}_{2}\right)$, al igual que en nuestro estudio.

Algunos hallazgos de nuestro estudio dignos de mencionar son los cambios citológicos de LIEBG o LIEAG que se observaron con mayor frecuencia en el grupo de solteras o divorciadas; las propias características de estas dos categorías podrían hacernos sospechar una mayor frecuencia de contactos sexuales ocasionales o menor probabilidad de tener una pareja fija, en ellas y en sus compañeros. Además, la mayoría de los casos de infección por VPH detectadas por captura de híbridos $\mathrm{HC}{ }^{\circledR}$ no mostraron alteraciones en la exploración ginecológica.

En contraste con la exploración ginecológica, en la citología sí se detectaron cambios inflamatorios inespecíficos; este hecho podría explicarse porque los cambios moleculares preceden a los cambios macro y microscópicos.

Nuestros datos muestran que la presencia de tipos de VPH de bajo riesgo oncogénico se detecta en mujeres con inicios más tempranos de la actividad sexual, con una edad media de 17 años y un intervalo de 13 a 21 años; mientras que en las pacientes infectadas con virus altamente oncogénicos, la edad media de inicio de su actividad sexual es de 19,5 años y un interva- lo de 14 a 34 años. Estos datos concuerdan con los publicados en la literatura.

En este sentido, nuestro estudio posee ciertas limitaciones, como el hecho de no haber dividido nuestra población en subgrupos de edad, lo cual tiene una relevancia importante si se compara con otros estudios publicados; además, el no haber incluido el tabaquismo como un factor asociado, pues está establecido como facilitador de progresión de infección por VPH al desarrollo neoplásico. El hecho de que solo se incluyera la captura de híbridos como técnica de diagnóstico molecular también limita el impacto de nuestro trabajo, ya que se ha demostrado que la reacción en cadena de la polimerasa (PCR) es más sensible y específica para VPH de alto y bajo riesgo. También se ha demostrado que la captura de híbridos es muy sensible, pero sólo modestamente específica como predictor de desarrollo de lesiones premalignas o malignas del cérvix uterino, ya que puede dar reacciones cruzadas entre ciertos tipos de VPH considerados de bajo riesgo.

En el presente trabajo, también hemos podido comprobar que la exploración ginecológica normal no descarta la presencia de VPH; igualmente, una citología normal o con cambios inflamatorios inespecíficos, tampoco permite concluir la ausencia de VPH. La detección temprana de infección por VPH requiere métodos moleculares de diagnóstico, por lo tanto podemos concluir que con los métodos tradicionales de diagnóstico, no es posible detectar todas las pacientes infectadas con VPH, por lo cual sería deseable practicar pruebas moleculares para diagnóstico de la infección.

\section{Declaración sobre conflicto de intereses}

Los autores declaran que no poseen conflictos de intereses.

\section{Agradecimientos}

Agradecemos el incondicional apoyo brindado al presente estudio por la Dra. med. Oralia Bar- 
boza Quintana, la Dra. med. Raquel Garza Guajardo y el Dr. Roger Adrián González Ramírez; sin los cuales no podríamos haber alcanzado el presente logro.

\section{Bibliografía}

1. ALWAN AD, MACLEAN D and MANDIL A. Assessment of national capacity for noncommunicable disease prevention and control: The report of a global survey. World Health Organization [WHO/MNC/01.2] 2001.

2. ZHANG WY, XUE YZ, CHEN $\mathrm{M}$, et al., Prevalence of high risk human papillomavirus infection in different cervical lesion among organized health-examination women in Shangai, China. Chin Med J 2008;121:1578-1582.

3. SIX L, LEODOLTER S, SINGS HL, et al., Prevalence of human papillomavirus types 6, 11,16 y 18 in young Austrian women -baseline data of a phase III vaccine trial. WienKlin Wochenschr 2008;120:666-671.

4. CERVANTES J, LEMA C, HURTADO L, et al., Prevalence of human papillomavirus infection in rural villages of the Bolivian Amazon. Rev Inst Med Trop. Sao Paulo 2003;45:131-135.

5. INEGI/Secretaría de Salud. Dirección General de Información en Salud. En Base de datos de defunciones 2005, México 2002.

6. TOVAR-GUZMÁN VJ, ORTIZ Contreras F, JIMÉNEZ Gauna FR, et al., Panorama epidemiológico de la mortalidad por cáncer cervicouterino en México (1980-2004). Rev Fac Med UNAM 2008;51:47-51.

7. ALWAN AD, MACLEAN D and MANDIL A. Assessment of national capacity for non communicable disease prevention and control: The report of a global survey. World Health Organization [WHO/MNC/01.2] 2001.
8. GARCÍA Tamayo J. MOLINA J. BLASCO Olaetxea E. El virus del papiloma humano y el cáncer cervical. Investigación Clínica. 51(2):193208, 2010 Jun.

9. ZURHAUSEN H. Human papilomaviruses and their possible role in squamouscell carcinomas. Curr Top Microbiol Immunol 1977;78:1-30.

10. JABLONSKA S, DABROWSKI J, JAKUBOWICZ K. Epidermodysplasia verruciformis as a model in studies on the role of papovaviruses in oncogenesis. Cancer Res 1972; 32:583-589.

11. LUTZNER MA. Epidermodysplasia verruciformis. An autosomal recessive disease characterized by viral warts and skin cancer. A model for viral oncogenesis. Bull Cancer 1978; 65:169-182.

12. SÁNCHEZ-ALEMÁN MA, URIBE-SALAS F, CONDE-GONZÁLEZ CJ. Human papillomavirus infection, a possible biological marker of sexual behavior among university students. Salud Publica Mex 2002;44:442-447.

13. FECHITA G, NEISES A. Task force consens report on HPV-related changes of the lower female genital tract. Acta Cytol 2002;46:630-632.

14. MOUGIN C, DALSTEIN V, PRÉTET JL, et al., Epidemiology of cervical papillomavirus infections. Recent knowledge. Press Med 2001;30:1017-1023.

15. KURMAN RJ, JENSON AB, LANCASTER WD. Papillomavirus infection of the cervix. II. Relationship to intraepithelial neoplasia based on the presence of specific viral structural proteins. Am J Surg Pathol 1983;7:39-52.

16. KJAER SK, VAN DEN BRULE AJ, PAULL $\mathrm{G}$, et al., Type specific persistence of highrisk human papillomavirus (HPV) as indicator of high grade cervical squamous intraepithelial lesions in young women: population based prospective follow up study. BMJ 2002;325:572-578. 